\title{
A DEVELOPMENT OF A POPPET TYPE BRAKE PRESSURE CONTROL VALVE FOR A FRICTION BRAKE OF ROLLING STOCK
}

\author{
Thum CHENVISUWAT*, Sung-Hwan PARK*, and Ato KITAGAWA* \\ * Department of Mechanical and Control Engineering \\ Tokyo Institute of Technology \\ 2-12-1 O-okayama, Mekuro-ku, Tokyo, 152-8552 Japan \\ (E-mail: kitagawa@ctrl.titech.ac.jp)
}

\begin{abstract}
A poppet type brake pressure control valve, which the output pressure is proportional to the input displacement, is developed to use in the hydraulic control system for friction brake of railway rolling stock. Internal feedback structure is designed to eliminate the reliability problem of the pressure transducer, and the poppet type valve is utilized to reduce the internal leakage, which is a problem of the conventional control valve when the electrical supply is under failure. Compensation methods for the disadvantages of poppet valve and the stability are considered together with the structure of the valve. Static and linearized dynamic models of the valve are determined to study the effects and to decide the parameters of valve. The stability and performance of valve are studied and confirmed experimentally. The experimental results show that the response of output pressure is improved compared with the conventional pneumatic brake system.
\end{abstract}

\section{KEY WORDS}

Railway, Hydraulic brake, Pressure control, Poppet valve, Internal feedback

\section{NOMENCLATURE}

$A_{e}:$ change in the cross-section area of seat

$A_{s}:$ cross-section area of seat

$A_{\nu}:$ area of flow passage in poppet valve

$A_{x}:$ cross-section area of control rod

$b_{p}$ : viscous damping coefficient of damper

$C_{d}:$ discharge coefficient of flow

$d_{v}$ : diameter of seat

$F_{b l}$ : force of compensating spring

$F_{b l 0}:$ initial force of compensating spring

$F_{x}$ : operating force of control device

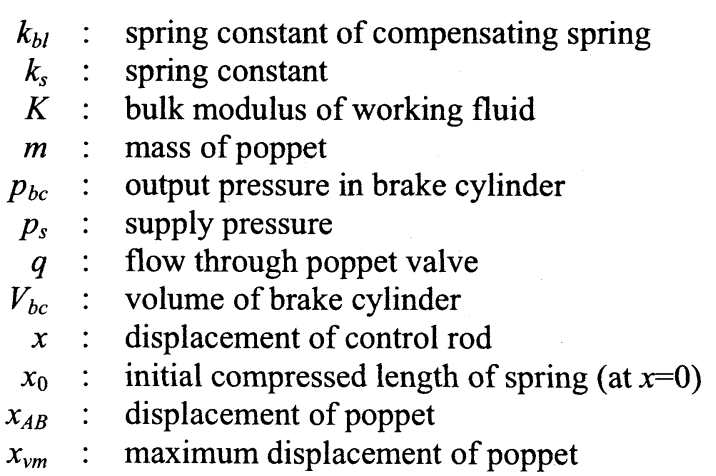




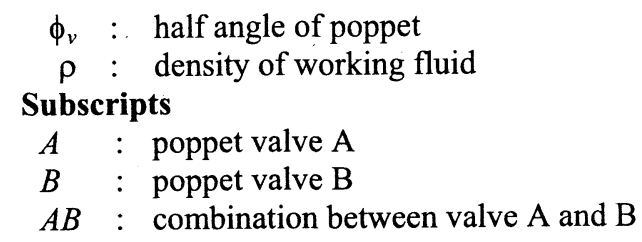

\section{INTRODUCTION}

The performances of two independent brake systems of modern railway, dynamic brake (electrical brake) and friction brake (mechanical brake), are derived. Our study concentrates on the control system of friction brake. The friction brake is an important device for the railway, since it is used as an auxiliary brake for the emergency situation, and also it is used to compensate the force and response of dynamic brake at both high and low speed regions. Recently, though the pneumatic system that controls the friction brake was developed into the electro-pneumatic system and the efficiency of brake system can be progressively improved by computer control system [1-3], the electro-pneumatic brake still has a lot of weak points such as a large size, a limitation of maximum pressure, and compressibility of air.

Since the use of high-pressure compact hydraulic control system could improve the response of brake system and reduce the overall weight of brake devices [4-5], we intend to apply it in the actual railway. It is found that the reliability of brake system also can be improved by installing the compact hydraulic brake units on each bogie. However, due to some application problems, till now the compact hydraulic brake has not been applied in any actual high-speed railway. Because of the internal leakage of pressure control valve, the compact hydraulic system that does not have the large tank cannot adequately generate braking force for the required time when the main electrical power supply fails. In addition, the possibility that the pressure transducer in pressure feedback control system might be damaged under the savage environment of railway is also a problem for the actual application.

Following to the above problems, it can be considered that the usages of a non-internal-leakage pressure control valve and a control system that can work without pressure transducer are desirable for the compact hydraulic system of railway. In this paper, a poppet type brake pressure control valve is proposed, in which the pressure of brake cylinder can be controlled by combining this valve with a displacement control device. Static and dynamic characteristics of the proposed valve are analyzed, and the experimental verifications are performed to confirm the validity of valve.

\section{BRAKE PRESSURE CONTROL VALVE}

Figure 1 shows a schematic diagram of the proposed poppet type brake pressure control valve (abbreviated by BPC valve). Two poppet valves are utilized to reduce the internal leakage and contamination sensitivity problem of conventional spool type brake pressure control valve. To assure the stability of valve, the flows of both poppet valves are designed in the divergence direction, and the internal dampers are put onto the poppets. The output pressure $p_{b c}$ to brake cylinder can be controlled by changing the position of control rod $x$, which relates to the pushing force on two poppets. The internal feedback structure is designed to eliminate the requirement for a pressure transducer.

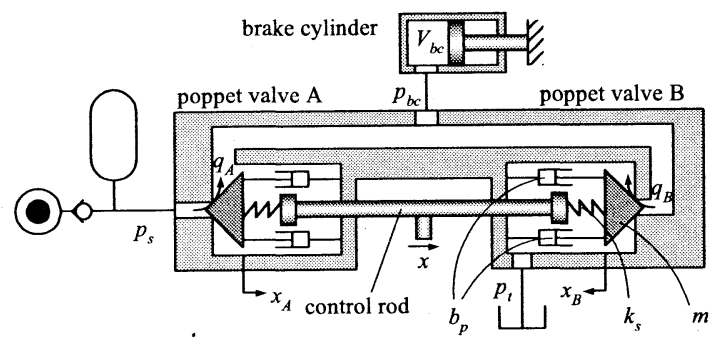

Figure 1 Brake pressure control valve

The working principle of BPC valve can be described as follows. In the steady-state condition, the closing forces by springs are greater than the opening forces by pressure; therefore, both of poppet valve A and B are closed. When the displacement of control rod $x$ is increased, the closing force by spring at valve $\mathrm{A}$ is reduced, then valve $\mathrm{A}$ is opened and output pressure increases. At the same time, the closing force by spring at valve $B$ is increased, so that valve $B$ is still closed as it was. While the output pressure increases, the area of flow passage at valve A is gradually reduced, and finally closed when the output pressure is above the set pressure. On the other hands, if the displacement of control rod $x$ is decreased, the set pressure is less than the output pressure, then valve $B$ is opened and the output pressure decreases.

\section{STATIC CHARACTERISTICS}

\section{OUTPUT PRESSURE}

The output pressure of the BPC valve can be determined by considering the balance of forces on the poppets. At the steady-state condition $\left(x_{A}=x_{B}=0, q_{A}=q_{B}=0\right)$, the closing condition of valve $\mathrm{A}$ and $\mathrm{B}$ can be expressed as 


$$
\begin{aligned}
& p_{b c}>\frac{\left(k_{s}+\Delta k_{s A}\right)}{A_{s}} x-\frac{\left(k_{s}+\Delta k_{s A}\right) x_{0 A}-p_{s} A_{s}}{A_{s}} \\
& p_{b c}<\frac{\left(k_{s}+\Delta k_{s B}\right)}{A_{s}} x+\frac{\left(k_{s}+\Delta k_{s B}\right) x_{0 B}}{A_{s}}
\end{aligned}
$$

where $\Delta k_{s A}, \Delta k_{s B}$ are the uncertainties of spring constants at valve $\mathrm{A}$ and $\mathrm{B}$.

From Eqs. (1) and (2), if we assume that the characteristics of springs at valve A and B are identically equal and linear $\left(\Delta k_{s A}=\Delta k_{s B}=0\right)$, then the closing condition of the BPC valve can be written as

$$
\frac{k_{s}}{A_{s}} x-\frac{k_{s} x_{0 A}-p_{s} A_{s}}{A_{s}}<p_{b c}<\frac{k_{s}}{A_{s}} x+\frac{k_{s} x_{0 B}}{A_{s}}
$$

In Eq. (3), the spring constant $k_{s}$, the cross-section area of seat $A_{s}$, the initial displacements of springs $x_{0 A}$, $x_{0 B}$, and the supply pressure $p_{s}$ are constant. Hence, it can be considered that the output pressure $p_{b c}$ will be in a boundary, which is approximately proportional to the displacement of control rod $x$.

\section{DEAD-BAND}

The output pressure in Eq. (3) can be written as

$$
p_{b c}=\frac{k_{s}}{A_{s}} x+\Delta p_{b c}
$$

where the error of output pressure $\Delta p_{b c}$ is given by

$$
-\frac{\left(k_{s} x_{0 A}-p_{s} A_{s}\right)}{A_{s}}<\Delta p_{b c}<\frac{\left(k_{s} x_{0 B}\right)}{A_{s}}
$$

The range of the error $\Delta p_{b c}$ can be considered as a width of dead-band characteristic in which the pressure cannot be controlled. This is the same as the effect of overlap in the spool valve. In order to reduce the leakage and the vibration at the steady-state condition, a small dead-band in the output pressure is desirable.

\section{OPERATING FORCE}

Since the fail-safe feature is required when designing a brake control system of railways, the operating force of control valve should be designed as low as possible to make sure that the output pressure can be generated and maintained by the backup power source. However, the unbalance of the forces due to the pressure and spring at control rod of the BPC valve might lead to a large operating force that is not preferred in the electric-power failure situation.

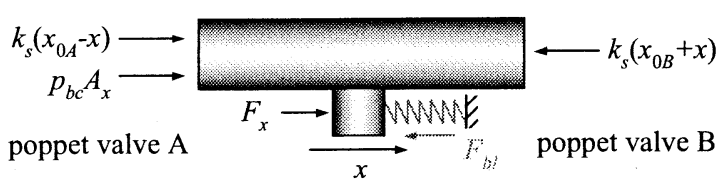

Figure 2 Forces acting on the control rod

The compensating spring as shown in Figure 2 is utilized to reduce the operating force. The forces acting on the control rod can be expressed as Eq. (6).

$$
p_{b c} A_{x}+k_{s}\left(x_{0 A}-x\right)+F_{x}=k_{s}\left(x_{0 B}+x\right)+F_{b l}
$$

where the friction and other forces are omitted for simplicity.

In order to determine the parameter of compensating spring, the dead-band of BPC valve is assumed to be adequately small $\left(\Delta p_{b c} \rightarrow 0, x_{0 A} \rightarrow p_{s} A_{s} / k, x_{0 B} \rightarrow 0\right)$. Using Eq. (4) and Eq. (6), the acting force on the control rod can be written as

$$
\begin{aligned}
& F_{x}=F_{x}^{\prime}+F_{b l} \\
& F_{x}^{\prime}=-\left(A_{x} / A_{s}-2\right) k_{s} x-p_{s} A_{s} \\
& F_{b l}=k_{b l} x+F_{b l 0}
\end{aligned}
$$

where $F_{x}^{\prime}$ is the operating force without compensation, and the spring constant of compensating spring $k_{b l}$ and the force due to the initial displacement of compensating spring $F_{b l 0}$ can be determined by minimizing Eq. (7).

\section{RELIABILITY IMPROVEMENT}

A disadvantage of poppet valve is that poppet and seat are subject to wear. This wear will lead to the changes on the initial displacement and cross-section area of poppet valve. For the BPC valve, it can be considered that the effect of the change on displacement can be compensated by re-adjust the initial displacement of spring. However, the effect of the change of cross-section area that will change the static characteristic of the BPC valve is not easy to compensate.

In this study, the compensation method is determined by analyzing the static characteristics of two structures. In case 1 , the angle of seat is designed to be less than the angle of poppet, as shown in Figure 3(a). In case 2, the angle of seat is designed to be greater than the angle of poppet, as shown in Figure 3(b). We found that in order to improve the reliability of BPC valve by reducing the effects of wear, the poppet valve $A$ should be designed as in case 1 , and the poppet valve B should be designed as 
in case 2.

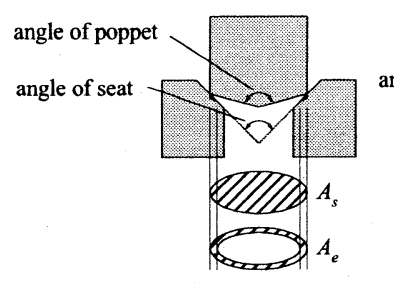

(a) Case 1

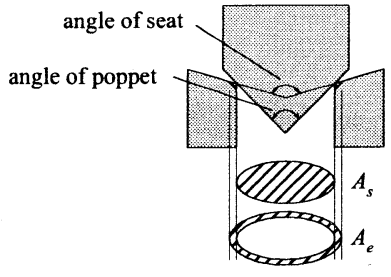

(b) Case 2
Figure 3 Relation between the angles of poppet and seat

\section{DYNAMIC CHARACTERISTICS}

In order to improve the transient response and stability of BPC valve, the dynamic characteristics and effects of parameters are analyzed. Because the BPC valve is composed of two poppet type valves, the relationship between the displacement of two poppets and the nonlinear characteristics of flows are complicated compared with the spool type valve $[5,6]$. In this chapter, simplified transfer functions of BPC valve are determined.

\section{FUNDAMENTAL MODEL}

The fundamental equations for the BPC valve can be expressed as follows. Using the equations of motion, the dynamics of the displacement of poppets can be expressed by

$$
\begin{aligned}
& m \ddot{x}_{A}+b_{p} \dot{x}_{A}+k_{s}\left(x_{A}+x_{0 A}-x\right)=\left(p_{s}-p_{b c}\right) A_{s}-F_{f A} \\
& m \ddot{x}_{B}+b_{p} \dot{x}_{B}+k_{s}\left(x_{B}+x_{0 B}+x\right)=p_{b c} A_{s}-F_{f B}
\end{aligned}
$$

where the cross-section area of seat $A_{s}=\pi d_{v}^{2} / 4$, and the flow force $F_{f A}$ and $F_{f B}$ are given by

$$
\begin{aligned}
& F_{f A} \approx C_{d} \pi d_{v} x_{A} \sin 2 \phi_{\nu}\left(p_{s}-p_{b c}\right)+\rho L \dot{q}_{A} \\
& F_{j B} \approx C_{d} \pi d_{v} x_{B} \sin 2 \phi_{v}\left(p_{b c}\right)+\rho L \dot{q}_{B}
\end{aligned}
$$

The pressure-flow characteristics of each poppet valve can be determined by

$$
q_{A}=C_{d} \pi d_{v} \sin \phi_{v} x_{A} \sqrt{\frac{2}{\rho}\left(p_{s}-p_{b c}\right)}
$$

$$
q_{B}=C_{d} \pi d_{v} \sin \phi_{v} x_{B} \sqrt{\frac{2}{\rho} p_{b c}}
$$

where $x_{A}<<d_{v}, x_{B}<<d_{v}$. Because the cross-section area of seat limits the maximum flow through the valves, the boundary conditions $0 \leq x_{A} \leq x_{v m}$ and $0 \leq x_{B} \leq x_{v m}$ are utilized in the mathematical model for these limitations.

The dynamics of output pressure in the brake cylinder can be expressed as

$$
\dot{p}_{b c}=\frac{K}{V_{b c}}\left(q_{A}-q_{B}\right)
$$

\section{LINEARIZATION}

In order to express the dynamic characteristics of output pressure and displacement of poppet by transfer functions, the nonlinear fundamental model is linearized. In this study, we considered that two valves are closed and there is no flow at the steady-state condition. By applying Taylor series around the operating point at $x_{A}{ }^{*}=0, x_{B}{ }^{*}=0, q_{A}{ }^{*}=0, q_{B}^{*}=0, p_{b c}{ }^{*}=p_{s} / 2$, and neglecting the higher order terms, the linearized equations can be determined.

We found that when equations for the poppet valve $\mathrm{A}$ and $B$ are combined together, the saturation characteristics of the displacements of poppet A and B can be combined. Therefore, valve $A$ and valve $B$ are assumed not to open at the same time, and the combined displacement $x_{A B}$ and flow $q_{A B}$ are defined as follows.

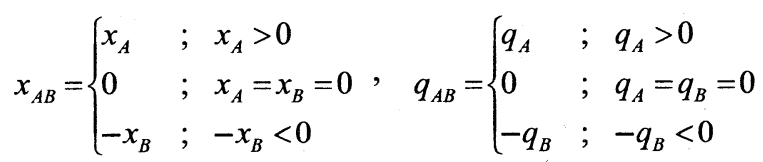

Using the above definitions, equation of motion, flow equation, and continuity equation can be written as

$$
\begin{aligned}
& k_{s} x-p_{b c} A_{s}=m \ddot{x}_{A B}+b \dot{x}_{A B}+k x_{A B} \\
& q_{A B}=C_{d} \pi d_{v} \sin \phi_{v} \sqrt{\frac{p_{s}}{\rho}} \cdot x_{A B} \\
& \dot{p}_{b c}=\frac{K}{V_{b c}} q_{A B}
\end{aligned}
$$

where the boundary condition of combined displacement 
of poppet is $-x_{v m} \leq x_{A B} \leq x_{v m}$, and the equivalent spring constant $k$ and damping coefficient $b$ are given by

$$
\begin{aligned}
& k=k_{s}+c_{d} \pi d_{v} \sin 2 \phi_{v} \frac{p_{s}}{2} \\
& b=b_{p}+\rho L c_{d} \pi d_{v} \sin \phi_{v} \sqrt{\frac{p_{s}}{\rho}}
\end{aligned}
$$

\section{TRANSFER FUNCTION}

Using Eq. (17), (18), (19), the simplified transfer function from the reference pressure $\tilde{p}_{b c}=\left(k_{s} / A_{s}\right) x$ to the output brake pressure $p_{b c}$ can be written as

$$
\frac{p_{b c}}{\widetilde{p}_{b c}}=\frac{\left(G_{1} / m\right)}{s^{3}+(b / m) s^{2}+(k / m) s+\left(G_{1} / m\right)}
$$

and the transfer function from the reference pressure to the displacement of poppets $x_{A B}$ can be written as

$$
\frac{x_{A B}}{\widetilde{p}_{b c}}=\frac{\left(A_{s} / m\right) \cdot s}{s^{3}+(b / m) s^{2}+(k / m) s+\left(G_{1} / m\right)}
$$

where $G_{1}=\frac{\pi^{2}}{4} C_{d} \sin \phi_{v} K \sqrt{\frac{p_{s}}{\rho}} \frac{d_{v}{ }^{3}}{V_{b c}}$.

By considering step response, we can understand that the convergence value of output pressure is equal to the reference pressure $\tilde{p}_{b c}$, and the convergence value of the displacement of poppet is 0 . In addition, we also can roughly understand the effects of parameters from these transfer functions. For example, if we design gain $G_{1}$ to have a large value, the response of output pressure can be improved. However, as the stability might be decreased, the appropriate parameters are necessary against this trade-off relation.

\section{EXAMPLE OF APPLICATION}

In this study, we use the simplified transfer functions Eqs. (22), (23) and a pole placement method to decide the main parameters of valve. A target settling time of output pressure in response to step input is designed to be 10 times faster than the conventional pneumatic brake $(0.05 \mathrm{~s})$, and the maximum displacements of poppets are designed to be less than $1 \mathrm{~mm}$ as shown in Figure 4; hence, the poles of transfer functions are designed at -80 , $-440,-440$, and the parameters are shown in Table 1 . It should be noted that these designed parameters will be adjusted later using numerical simulation, and considering valve characteristics and results of experiments.
Table 1 Designed parameters of the BPC valve

\begin{tabular}{rlr|rlr|}
$p_{s}$ & $=12$ & $\mathrm{MPa}$ & $m$ & $=0.54$ & $\mathrm{~kg}$ \\
$C_{d}$ & $=0.6$ & - & $b$ & $=518$ & $\mathrm{~kg} / \mathrm{s}$ \\
$K$ & $=1.58 \times 10^{3}$ & $\mathrm{MPa}$ & $k$ & $=143 \times 10^{3}$ & $\mathrm{~N} / \mathrm{m}$ \\
$V_{b c}$ & $=3.54 \times 10^{-4}$ & $\mathrm{~m}^{3}$ & $d_{v}$ & $=4 \times 10^{-3}$ & $\mathrm{~m}$ \\
$\rho$ & $=870$ & $\mathrm{~kg} / \mathrm{m}^{3}$ & $\phi_{v}$ & $=0.17$ & $\mathrm{rad}$ \\
\hline
\end{tabular}

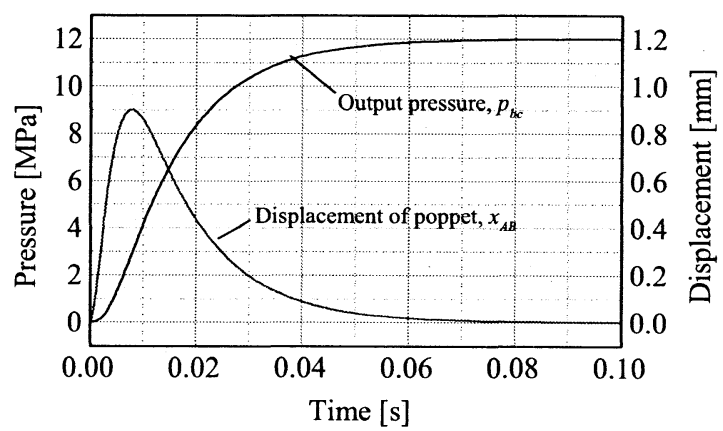

Figure 4 Step response (Simulation)

\section{EXPERIMENTS}

The experimental devices for testing the characteristics of BPC valve are set up as shown in Figure 5. In this experiment the displacement of control rod is controlled by servo cylinder. To study the stability of valve, two cylinders connected in series are used instead of the actual friction brake device (caliper brake). The volume of pressure control chamber can be controlled using a computer. The volume is set at about $40 \mathrm{~cm}^{3}$, which is same as the real brake device of Shinkansen, and small disturbance is inserted.

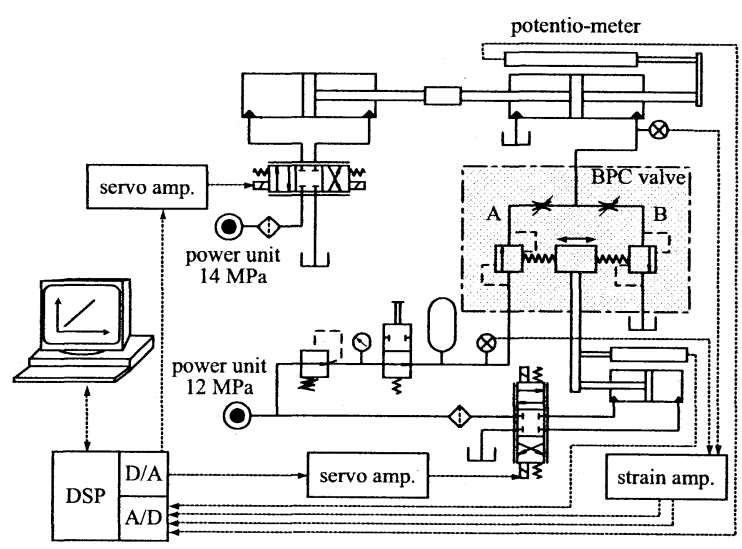

Figure 5 Experimental set-up 
The step up and step down responses of output pressure for each command displacement are shown in Figure 6. The results show that relationship between the displacement of control rod and output pressure is approximately linear. For the tested range of pressure, the response is adequately fast and stable.

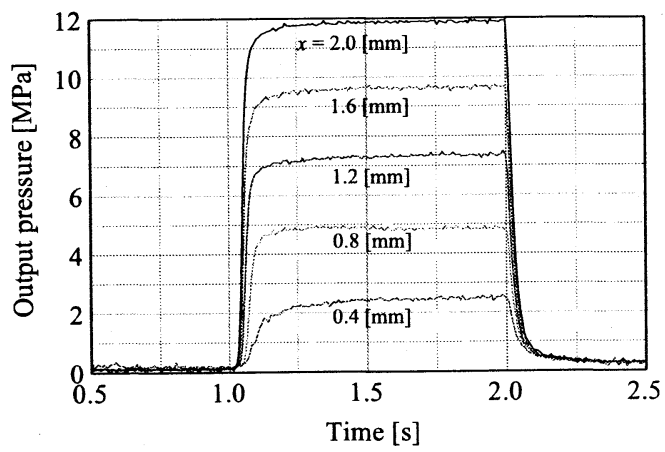

Figure 6 Step input experimental results

Figure 7 shows the frequency response of pressure control using the actual caliper brake device of Shinkansen. The system can correspond to the command pressure well up to $1 \mathrm{~Hz}$, after that the gain becomes smaller and the phase delay occurs. The phase delay becomes 90 degrees at the frequency of about $50 \mathrm{~Hz}$.
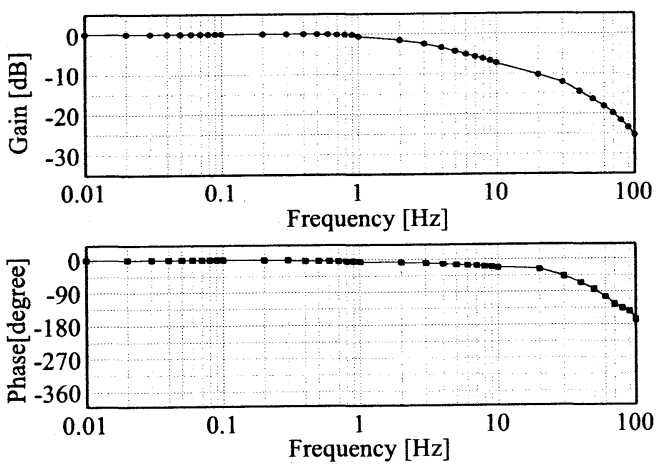

Figure 7 Frequency response using caliper brake

Figure 8 shows the comparison of a hydraulic brake system using the BPC valve, a hydraulic brake system using High Speed Solenoid Valves (HSSV) [4], and a conventional pneumatic brake system. The results show that the response of the hydraulic brake is much faster than the pneumatic brake. Because the flow of BPC valve is smaller when the error of output pressure is smaller, the rising time of pressure control using the BPC valve is little slower than the pressure control using HSSV. However, it is found that the response of output pressure of the BPC valve can be improved, if we increase the loop-gain in Eq. (22) and adjust other parameters for the stability.

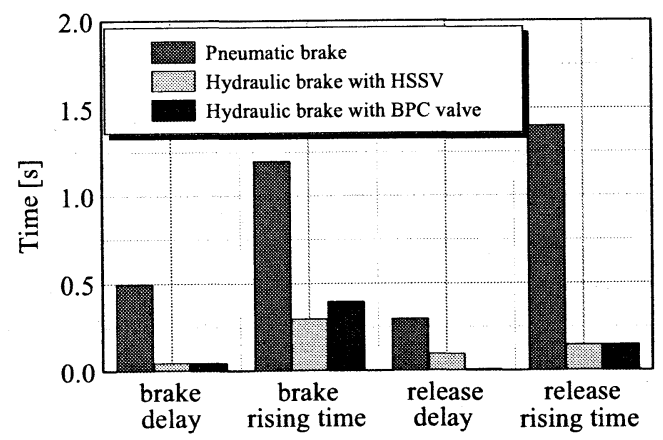

Figure 8 Comparison of brake pressure control response

\section{CONCLUSION}

To develop a small size hydraulic brake system for the railway, the poppet type brake pressure control valve (BPC valve) was proposed to solve the internal leakage problem of conventional control valve together with the reliability problem of pressure transducer. The static characteristic of the proposed valve was analyzed, the operating force of displacement control device was compensated, and the method to improve the performance against the wear was determined. In order to improve the dynamic performance of the BPC valve, the simplified transfer function was determined, and the effects of parameters were analyzed. The experimental results confirmed the validity of valve, and clarified that the response of brake pressure control can be improved by hydraulic system using the BPC valve.

\section{REFERENCES}

1. A.C. Sharp, Railway braking: a history, Engineering, 1979, 219-3, pp.1112-1122

2. J Leigh, Development of modular locomotive brake systems, IMechE, 1996, 2001-18, pp.97-106

3. J E Paddison, W P Straube, Advanced modular EP brake control, IMechE, 2000, 2001-4, pp.259-265

4. S. Uchida, N. Kumagai, Development of Hydraulic Brake for Rolling Stock, RTRI report, 1998, 12-1, pp.1-6 (in Japanese)

5. T. Kaneko, A Study on Brake Equipments for Rolling Stock, Trans. of the JSME, 1978, 44-379, pp.907-915 (in Japanese)

6. T. Ichikawa, K. Nakamura: Stability of Hydraulic Poppet Valve, Trans. of the JSME, 1968, 34-257, pp.91-99 (in Japanese) 